\title{
Intelligent Control of Metal Filling by Welding Robots
}

\author{
Wei $\mathrm{Fu}^{1, \mathrm{a}^{*}}$, Jiajia $\mathrm{Ni}^{2, \mathrm{~b}}$, Chengxian Zhou ${ }^{3, \mathrm{c}}$, Yan Chen ${ }^{4, \mathrm{~d}}$ \\ 1,2,3,4 Xiamen University of Technology,600,LiGong Rd,JiMei,XiaMen, \\ Postcode 361024 ,China \\ afuwei@xmut.edu.cn,,rgnijiajia@163.com, ${ }^{\mathrm{c}}$ zhouchengxian@xmut.edu.cn, \\ dchenyan@xmut.cn
}

Keywords: Welding robot, Kinematics inverse problem ,Binocular stereo vision camera, Metal filling, Laser array sensor.

Abstract: robot model of three-arm and five-degree freedom plus large scope of traversing welding was established, and decoupling of models of "large scope of traversing", "triangle movement of two arms" and "spherical movement of one arm" was realized. The model of "triangle movement of two arms "is able to use geometrical calculation to solve the kinematics inverse problem, avoid the multiplicity, improve the calculation speed, eliminate the blind spots of the motions of welding gun of welding robot, and simplify the kinematic pair of kinematic mechanism for the arc filling strategy during welding travelling of robot. Binocular stereo vision camera was used to detect the edges of welds, and laser array sensor was used to measure the weld data. The matlab program is designed according to the shape of weld to calculate the amount of metal filling. Relevant date has been used to calculate the accuracy of the percent and analyze the factors that lead to the errors.

\section{Introduction}

Now robots with six-degree freedom structure are applied in both domestic and foreign markets, whose critical defect is that the inverse problem of kinematics must be solved by inversion of homogeneous matrix. This calculation is slow and has multiple solutions. In particular, when the homogeneous transfer matrix is singular, the inversion problem of kinematics has no solution[1,2]. That is to say, welding robots with six-degree freedom structure used in open environment have blind spots and the solution accuracy of inversion problem of kinematics nearby the blind spots is greatly reduced. Therefore, three-arm and six-degree freedom welding robots in the current market can only work in the mode of teaching box and cannot work in completely open environment. The welding robot during the plate welding usually weld with deviation and false action due to factors like strong arc light, high temperature, fume and error of workpiece, clamp accuracy, surface conditions and thermal deformation of workpiece. This is bottleneck where current three-arm and six-degree freedom welding robots cannot be widely applied to industries[3,4].

In open operation conditions, two basic conditions must be addressed for welding robots. Firstly, sensor which can observe the open operation condition; at the second place, the robot can be controllable in completely open operation conditions. In order to empower the robot with the functions to observe the environmental condition and coordinates of target position, perform the analysis and judgment, and finish the tasks intelligently and independently, binocular stereo vision system was adopted in this paper to be used as the guide of tracking control of arc welds. Within the small scope, laser array was used to check accurately the three-dimensional shape of the welds, 
track in real time the position, size, concave and convex faces of the work piece to be welded, select intelligently the filling strategy of the welding robot, plan in real time the trajectory and the metal filling amount of the welding robot. Finally during the high-temperature welding, the size of stem extension of arc sensors of electric three-arm and five-freedom plus large scope of traversing was used to control the swinging of the welding robot so that the robot could be widely applied to work in open environment. In order to be controlled in open work conditions, special mechanism of "welding robot of three-arm and five-degree freedom plus large scope of traversing whose models can be decoupled" was established in this paper. It could be decoupled into the superposition of the model of "large scope of traversing" and the model of "three-arm and five-degree freedom mechanic arm of welding robot", while the model of "three-arm and five-freedom mechanic arm of welding robot" was further decoupled into the superposition of model of "triangle movement of two arms" and the model of "spherical movement of one arm". The model of "triangle movement of two arms" could use geometrical calculation to solve the inverse problem of kinematics to avoid multiplicity, increase the calculation speed, eliminate the blind spots of motions of welding gun of the welding robot, and simplify the kinematic pair of kinematic mechanism for arc filling strategy during welding travelling of the robot. The model of "spherical movement of one arm" and the model of "large scope of traversing" were simply the translation relation.

\section{Robot Structure of Three-arm and Five-degree Freedom Plus Large Scale of Traversing}

As shown in Fig1, this kind of robot is defined in accordance with six Cartesian coordinates $\{\mathrm{k}\}$; $\mathrm{k}$ is from 0 to 5 . Obviously when traversing in large scope the $\{0\}$ coordinate, only the direction of $x 0$ is traversed in large scale, so it is enough to let yl traverse the welding robot in the opposite direction in coordinate of $\{1\}$. Therefore, the direct problem of kinematics of this kind of robot only considers the mapping transformation of the three-arm and five-freedom manipulator.

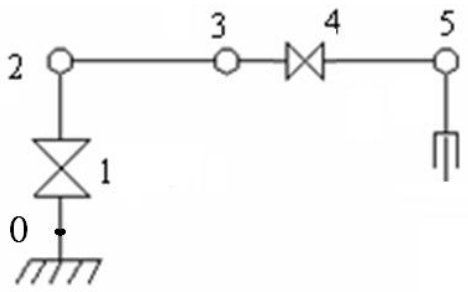

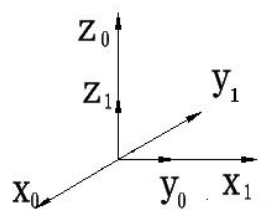

$\{0\}\{1\}$

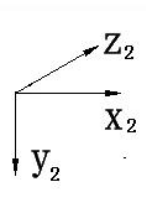

$\{2\}$

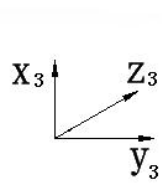

$\{3\}$

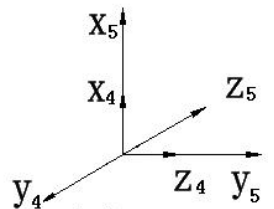

$\{4\}\{5\}$

Fig.1. six Cartesian coordinates for welding robots of three-arm and five-degree freedom plus large scope of traversing

The attitude at the end of the three-arm and five-degree freedom mechanic arm of the welding robot can be described with five independent variables: $\left[\begin{array}{lllll}\theta_{1} & \theta_{2} & \theta_{3} & \theta_{4} & \theta_{5}\end{array}\right]^{T}$. The direct problem of kinematics of five-degree freedom welding robot can be transformed with formula (1.1) for the direct problem of kinematics of mechanic arm described by Denavit-Hartenberg method.

$$
{ }_{5}^{0} \boldsymbol{T}={ }_{1}^{0} \boldsymbol{T}\left(\theta_{1}\right){ }_{2}^{1} \boldsymbol{T}\left(\theta_{2}\right){ }_{3}^{2} \boldsymbol{T}\left(\theta_{3}\right){ }_{4}^{3} \boldsymbol{T}\left(\theta_{4}\right){ }_{5}^{4} \boldsymbol{T}\left(\theta_{5}\right)
$$

If the formula (1.1) for direct problem of kinematics of five-degree freedom welding robot is solved inversely, the joint variables of $\left[\begin{array}{lllll}\theta_{1} & \theta_{2} & \theta_{3} & \theta_{4} & \theta_{5}\end{array}\right]^{T}$ corresponding to attitude at one end of the welding robot can be obtained. This kind of inverse problem of kinematics must be solved 
through inversion of transformation matrix of each connection rod, in which the calculation speed is slow and it has multiple solutions. In particular, when the homogenous transfer matrix is singular, the inverse problem of kinematics has no solution. That is to say, it is still not enough to obtain the joint variables by direct inversion of formula (1.1) for positive problem of kinematics of five-degree freedom welding robot. How to solve this difficult problem was one key issue of this paper.

As shown in Fig.1, when the welding robot traverses in large scale the travelling mechanism in $\{0\}$ coordinate, only the direction of xo is traversed in large scale. So it is enough to let y1 traverse in opposite direction the welding robot with relevant transverse length in coordinate $\{1\}$.

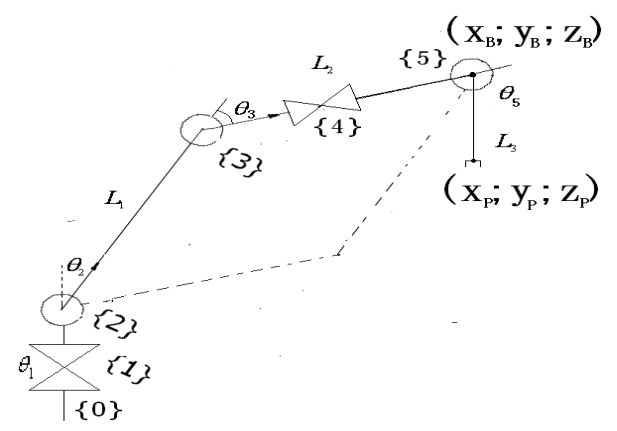

Fig.2. Coordinate corresponding to attitude of end position of weld

Assume that in Cartesian coordinate $\{1\}$, given the corresponding coordinate $\left\{\mathrm{x}^{\mathrm{p}}, \mathrm{y}^{\mathrm{p}}, \mathrm{z}^{\mathrm{p}}\right\}$ of attitude at one end of the welding robot, when it is required that the trajectory of welding robot keep not shaking during the welding and the welding gun keep perpendicular to the welding surface, the $\left\{\mathrm{x}^{\mathrm{B}}, \mathrm{y}^{\mathrm{B}}, \mathrm{z}^{\mathrm{B}}\right\}$ and the coordinate corresponding to welding point only traverse with the rod length $\mathrm{L}$ in the direction z1 in Cartesian coordinate. During the design of welding robot of three-arm and five-degree freedom plus large scale of traversing, the joint angle is made larger than 0 through limit mechanism, that is to say, the three-arm and five-degree freedom mechanic arm of this welding robot will not have the attitude at the welding end related to the dotted line shown in Fig 2.

The expected attitude of three-arm welding robot corresponding to welding point $\mathrm{P}$ can be fixed through above three methods. By triangular geometrical calculation, we have:

$$
\begin{aligned}
& \theta_{1}=\arg \tan \left(y_{P} / x_{P}\right) \\
& \theta_{2}=\frac{\pi}{2} \arg \tan \left(\left(z_{p}+L_{3}\right) / \sqrt{x_{p}^{2}+y_{p}^{2}}\right)-\operatorname{argcos}\left(\frac{x_{p}{ }^{2}+y_{p}{ }^{2}+\left(z_{p}+L_{3}\right)^{2}+L_{1}{ }^{2}-L_{2}^{2}}{2 L_{1} \sqrt{x_{p}^{2}+y_{p}^{2}+\left(z_{p}+L_{3}\right)^{2}}}\right) ; \\
& \theta_{3}=\pi-\arg \cos \left(\frac{L_{1}^{2}+L_{2}{ }^{2}-x_{p}{ }^{2}-y_{p}{ }^{2}-\left(z_{p}+L_{3}\right)^{2}}{2 L_{1} L_{2}}\right) \\
& \theta_{4}=0 \\
& \theta_{5}=\pi-\theta_{2}-\theta_{3} ;
\end{aligned}
$$

Among which: $\theta_{1} 、 \theta_{2}, \theta_{3}, \theta_{4} 、 \theta_{5}$ are joint angles of free-degree freedom mechanical arm respectively; $L_{1}=480 \mathrm{~mm}, L_{2}=480 \mathrm{~mm}, L_{3}=280 \mathrm{~mm}$ are length of three arm rods respectively. 
The geometrical calculation of (1.2) solves the difficult problem of solution of inverse kinematics of welding robot of three-arm and five-degree freedom plus large scale of traversing. Assume that one end position( $\mathrm{x} ; \mathrm{y} ; \mathrm{z})$ of welding robot was given, the joint variables of $\left[\begin{array}{lllll}\theta_{1} & \theta_{2} & \theta_{3} & \theta_{4} & \theta_{5}\end{array}\right]^{T}$ related to the attitude can be quickly obtained by geometrical calculation (1.2) and this is one-to-one correspondence. The welding gun of the welding robot is able to reach the expected end position point only by controlling the five servo motors to these joint variables.

When the welding robot is required to work as human beings, which is to control the welding gun to make operations like up, down, left, right, stretch and extend in Euclidean three-dimensional $\operatorname{space}(\mathrm{x}, \mathrm{y}, \mathrm{z})$, it is just to make the welding robot in Cartesian coordinate $\{1\}$. Based on one end position of wielding robot and through the geometrical calculation of (1.2), joint angel variables of $\left[\begin{array}{lllll}\theta_{1} & \theta_{2} & \theta_{3} & \theta_{4} & \theta_{5}\end{array}\right]^{T}$ related to the attitude can be obtained and then the requirement can be fulfilled when the five position servo motors are controlled in to place. This kind of "linked operation" mode is line with the human welding habits.

As shown in Fig2, $\theta_{4}=0$, although $\theta_{5}$ is joint variable, rod $L_{3}$ always keeps parallel to ${ }^{Z_{1}}$. The welding gun with this attitude keeps unchanged during welding, which is equivalent to the fact that the gun is always perpendicular to the welding surface. In real welding conditions, the welding surface is not always parallel to the surfacbes of $x_{1} O_{1} y_{1}$. At this time, change $\theta_{4}$ from $-90^{\circ}$ to $90^{\circ}$ and aim at the welding surface. Particularly during fillet welding, the welding gun should be raised to certain angle in elevation direction, at which moment, with calculated joint variable of $\theta_{5}, \theta_{5}$ can be reduced by $\Delta$ from $0^{\circ}$ to $90^{\circ}$. Obviously to keep the welding gun not shaking, it is required that the $\theta_{4}$ and $\Delta$ keep unchanged during one welding.

As shown in Fig2: with basic conditions of $\theta_{4}=0$, and $\theta_{5}$ of joint variable, $\theta_{4}$ from $-90^{\circ}$ to $90^{\circ}$; with additional $\theta_{5}$ of joint variable, $\theta_{5}$ is reduced by $\Delta$ from $0^{\circ}$ to $90^{\circ}$ and the space diagram is shown in Fig 3,since $\theta_{4}$ and $\Delta$ are within the value scope, just like arm $L_{3}$ is moving under hemisphere, so it is called "model of one arm spherical movement".

Geometrical correspondence of this model is:

$$
\begin{aligned}
& \mathrm{x}_{B}=\mathrm{x}-L_{3} \bullet \sin \Delta \\
& \mathrm{y}_{B}=\mathrm{y}-L_{3} \bullet \cos \Delta \bullet \sin \theta_{4} . \\
& \mathrm{z}_{B}=\mathrm{z}-L_{3} \bullet \cos \Delta \bullet \operatorname{con} \theta_{4}
\end{aligned}
$$

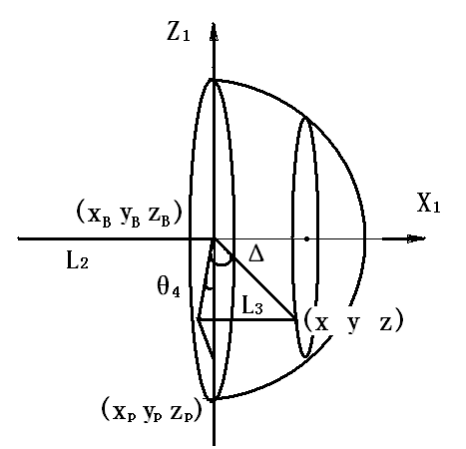

Fig.3. model of one arm spherical movement 


\section{Weld Tracking Technology of Welding Robots Based on Multi-sensor Data Fusion in Open Conditions}

Currently, sensors on welding robots which can reflect the site conditions for work pieces to be welded are one-axis high voltage contact sensor, arc sensor, laser sensor and weld tracking sensor for linear array CCD and two-dimensional area array CCD. The function of these sensors is to detect accurately the information of position and shape of welds and transform it into electric signals to be processed in real time by the control system. The control system controls the motor position servo mechanism based on the detection results, adjusts the attitude of welding gun, swinging strategy and metal filling amount, so as to realize automatic tracking of welds. However, the laser sensors, weld tracking sensors for linear array CCD and two-dimensional area array CCD picture cannot solve radically the problem of site condition feedback. Two sensing technologies for welding robots which are mostly applied and mature are: firstly, high voltage contract sensing: its function is also called initial position tracking technology. It is based on principles of locating before welding and on-line compensation, but the efficiency of the welding robots is greatly reduced. Besides, during the sensing, situations like wear of spare parts and bending of welding wire are factors which affect the accuracy. What is more, the application of non-contact sensor for arc weld tracking can correct on line the trace deviation of the robot. Generally, the arc weld tracking must trace with the initial point, so in combination with the contact sensing function, the welding current will be changed when the voltage of network is changed and contact tube is worn. Therefore, the welding deviation and error actions often occur on site, which is a great barrier for further application of plate wielding[5].

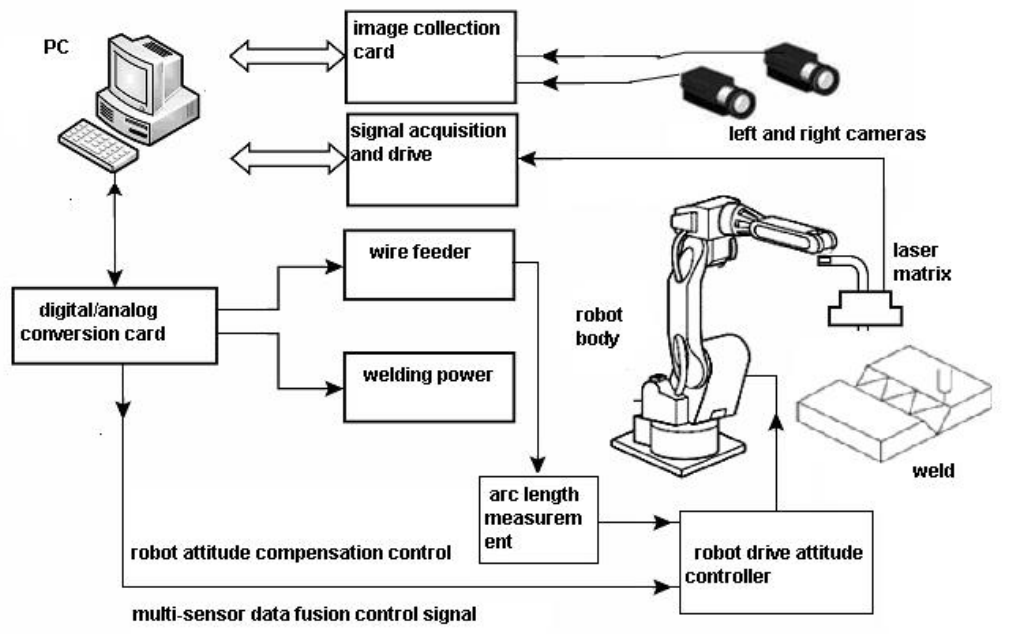

Fig.4. Overall structure of welding robot with multi-sensor data fusion

As shown in Fig4, this purpose of this research is to make the welding robots work in open environment (namely: inaccurate positioning of workpiece, irregular weld size and rough welding face). Binocular stereo vision camera was adopted in this paper to obtain on line the weld position coordinate $(\mathrm{x}, \mathrm{y}, \mathrm{z})$ of the work piece to be welded in open environment. Firstly in the large space scope, the left image $\mathrm{f}(m, n)$ collected by binocular stereo camera was used. It was assumed that left image $\mathrm{f}(m, n)$ was collected by binocular stereo camera and the storage format of the pixel was $360 \times 360$. Among which: $\mathrm{m}, \mathrm{n}$ were the line and row coordinate of the pixel; $\mathrm{f}(m, n)$ was the 
gray value corresponding to the coordinate. The assumption was that left image $\mathrm{f}(m, n)$ was collected by binocular stereo camera, the line coordinate of the pixel was in line with the direction of weld extension, and first order row difference was made at this time for grey value $\mathrm{f}(m, n)$ corresponding to the pixel coordinate, and then average grey value of $\mathrm{DV}=\frac{1}{360 \times 360} \sum_{m=1}^{360} \sum_{n=1}^{360} \partial \mathrm{f}(m, n)$ was gained for discretized points of the obtained row difference $\partial \mathrm{f}(m, n)$; then gray value of discretized points of row difference $\partial \mathrm{f}(m, n)$ was transformed as follows: $T_{L} \partial \mathrm{f}(m, n)=\left\{\begin{array}{cc}0 & \partial f(m, n) \leq D V \\ 255 & \partial f(m, n)>D V\end{array}\right.$, apparently the black area of image corresponding to discretized points function of $T_{L} \partial \mathrm{f}(m, n)$ was the left image obtained by binocular stereo vision camera. Finally, line coordinate information of pixels at edges of both weld ends was gained in turn in the direction of pixel increase of row coordinate for left image of weld $T_{L} \partial \mathrm{f}(m, n)$. The right and left images were matched by using the calibration of binocular stereo vision camera and formulate once again the space coordinate related to information points of edges of both weld ends $\left(x_{i}{ }^{1}, y_{i}{ }^{1}, z_{i}{ }^{1}\right)$ corresponding to upper weld curvature, $\left(x_{i}{ }^{2}, y_{i}{ }^{2}, z_{i}{ }^{2}\right)$ corresponding to lower weld curvature[6,7].

As shown in Fig4, laser array sensors are arranged in the radial direction of upper and lower weld curvatures. Six sensors in total with interval of $0.4 \mathrm{~mm}$, perpendicular to the welding surface are non-contact measurement with the measurement scope of $45 \mathrm{~mm}$ to $90 \mathrm{~mm}$. When welding robot of three-arm and five-degree freedom plus large scale of traversing is traversing in areas $45 \mathrm{~mm}$ above the middle line of upper and lower weld curvatures, the laser array sensor can obtain accurately the dimensions and three-dimensional shape of the welds so as to gain on-line in real time the volume of metal filing by welding robot. In particular, during the welding of plates, due to the large amount of metal filling, air holes, cracks and loose region will occur when the welding is finished in one time. Therefore, the metal filling has to be finished in several times so as to finish high-quality welding. In this condition, welding robots based on multi-sensor data fusion have more advantages. After the first welding, laser array sensors are used to detect the sizes and three-dimensional shape of the welds, and metal filling amount of next welding is planned in real time, thus avoiding the deviation due to deformation of high-temperature bath[8,9].

\section{Space Position Control System of Welding Robots with Attitude Compensation Feedforward in Open Conditions}

As shown in Fig4, the feedforward signals of attitude compensation for space position control system of welding robots are generated by weld tracking results of data fusion of multi-sensors. Firstly, the middle line of upper weld curvature $\left(x_{i}{ }^{1}, y_{i}{ }^{1}, z_{i}{ }^{1}\right)$ and lower weld curvature 
$\left(x_{i}^{2}, y_{i}^{2}, z_{i}^{2}\right)$ obtained by binocular stereo vision system is used as space travelling curvature of welding robot $\left(x_{p i}, y_{p i}, z_{p i}\right)$. Apparently with formula (1-2), five joint angle variables corresponding to attitude of $\left(x_{p i}, y_{p i}, z_{p i}\right)$ at the end of three-arm and five-freedom mechanism arm can be obtained quickly $\left[\begin{array}{llllll}\theta_{1 p i} & \theta_{2 p i} & \theta_{3 p i} & \theta_{4 p i} & \theta_{5 p i}\end{array}\right]^{T}$. Secondly, weld dimensions and three-dimensional shape obtained with laser array sensors are used as the estimate of metal filling amount for welding during space travelling of welding robot. Particularly in welding of plates, in order to increase the metal filling, the welding gun has to swing and advance. During swinging and advancing, large scale traversing mechanism of welding robot of three-arm and five-degree freedom plus large scale of traversing can be used to finish the advancing, and the joint variable of $\theta_{5}$ can be used to make circular swinging, which simplifies the kinematic pair of the robot. The travelling time is determined by current of robot and the circular swinging $\theta_{5}$ is determined by weld width.

As shown in Fig4, during welding, the welding robot might produce slight weld deformation due to high temperature. The binocular stereo vision and laser array sensor can not take effect due to the action of strong arc light. In this circumstance, extension electric acr sensor can be used to reflect in real time the slight deformation of welds[10].

By doing this, in open work conditions, with the space position feedback control of welding robot with attitude compensation feedforward control, the operation target of working in completely open conditions can be achieved.

"Welding robot of three-arm and five-degree freedom plus large scope of traversing whose models can be decoupled" could be decoupled into the superposition of the model of "large scope of traversing" and the model of "triangle movement of two arms" and the model of "spherical movement of one arm". The model of "triangle movement of two arms" could use geometrical calculation to solve the inverse problem of kinematics to avoid multiplicity, increase the calculation speed, eliminate the blind spots of motions of welding gun of the welding robot, and simplify the kinematic pair of kinematic mechanism for arc filling strategy during welding travelling of the robot. That is to say, during the weld tracking space, the newly established welding robots of three-arm and five-degree freedom plus large scope of traversing could be controlled anywhere in real time. The experiment proves that in completely open environment, the weld tracking accuracy can reach $0.1 \%$, meeting the welding process requirements.

Upper weld curvature $\left(x_{i}{ }^{1}, y_{i}{ }^{1}, z_{i}{ }^{1}\right)$ and the lower weld curvature $\left(x_{i}{ }^{2}, y_{i}{ }^{2}, z_{i}{ }^{2}\right)$ detected by binocular stereo vision camera were used, metal filling was detected by laser array sensors, real time welding control signals based on multi-sensor data fusion technology were formed. In completely open situations, space position control system for arc extension of welding robots with attitude compensation feedforward was realized, which apparently improves the weld success rate and welding quality. 


\section{Data Acquisition from Laser Array Sensor}

The photoelectric analog sensor of Contrinex can be used as laser array sensor, which has the advantages of diffuse reflection sensor. The measuring distance of the high precision laser analog sensor reaches $100 \mathrm{~mm}$, with the resolution of $40 \mathrm{um}$ in the range of $60 \mathrm{~mm}$, and the spot size of $3 \mathrm{~mm}$ in the $100 \mathrm{~mm}$ visible light. The main parameters of the sensor is the detection distance. The light emitting device and the photoelectric device are arranged in a certain direction in the same detection head. When there is a reflective surface (detected object) approaches, the photoelectric device receives the reflected light and output the signal, which can be perceived to be close to the object. The photoelectric analog sensor of Contrinex LAS/LAK-5050L-109 series can be used, and its measuring range is from $45 \mathrm{~mm}$ to $85 \mathrm{~mm}$, voltage output is from $0 \mathrm{~V}$ to $10 \mathrm{~V}$. Lower than $45 \mathrm{~mm}$ or higher than the limit $85 \mathrm{~mm}$, the servo power supply will be disconnected. Each $1 \mathrm{~V}$ represents $4 \mathrm{~mm}$, with a resolution of $0.02 \mathrm{~mm}$. Connection mode: Brown power cable can be connected to the DC power supply $28 \mathrm{~V}$ to $18 \mathrm{~V}$ as the working voltage, and the proposed power supply voltage stability is $24 \mathrm{~V}$ or so. Blue and black signal lines are grounded, and white is the signal output line.

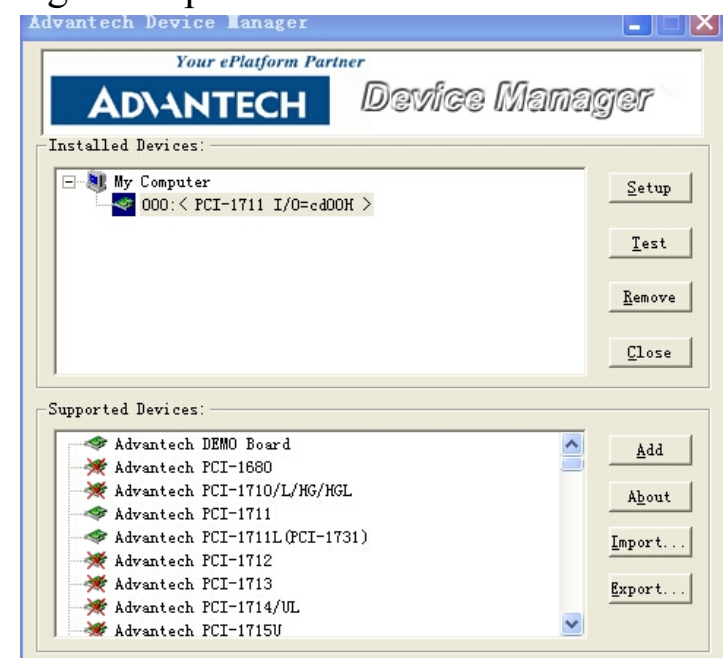

Fig.5. "Manager Device" dialog box

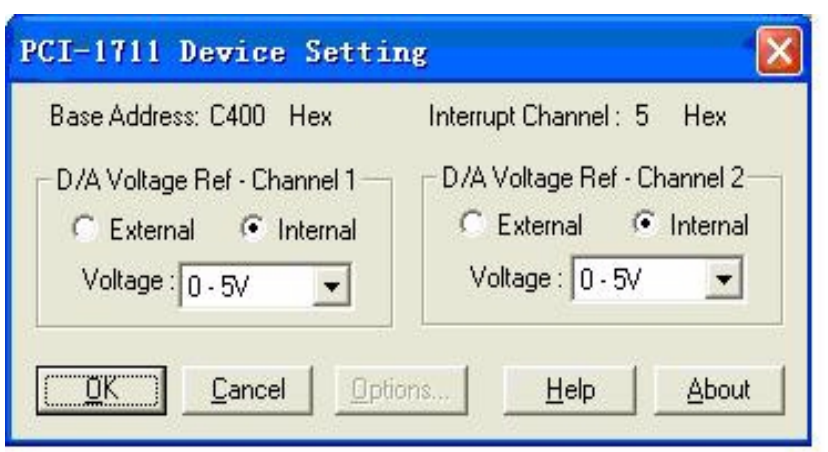

Fig.6. Setup dialog box

In order to detect to distance we need to convert analog signal into digital quantity. The Advantech PCI-1711 multifunction DAS card is installed in computer PCI card slot and the card contains 16 single-ended A/D input channel, accompanying with PCL-10168 cables and wiring ADAM-3968 wiring board. PCL-10168 shielded cable designed for the PCI-1711 card can provide a high degree of noise protection. In order to obtain the better signal quality, the signal cable is twisted to reduce the signal interference and the noise from other sources. In addition, its analog and digital cable are shielded to avoid the EMI/EMC problem. ADAM-3968 is a 68 pin SCSI terminal module for the installation of DIN guide rail. The terminal module can be installed on a card of Advantech PC-Lab, and the connection access of PCI-1711 card single pin is simple and reliable. White signal wire is connected to the ADAM-3968 terminal, and the terminal channel is selected from the AI input channel. The pin number is selected from 61 to 68 or 27 to 34, and the AIGND and DGND are also noted. PCL-10168 shielded cable is used to connect PCI1711 and ADAM-3968 connection board.

Drivers installed here have not much to say. Install drive in the start menu to find the "Manager Device" is shown in Fig.5. 
Click the "Setup" button to see the Setting Device dialog, as shown in Fig.6.

Set the reference voltage of the AO channel which is used internally or externally, if the internal reference voltage is used, $0-5 \mathrm{~V}$ or $0-10 \mathrm{~V}$ can be selected. After setting up, point OK button to save settings.

To do a test, in the normal working condition of the photoelectric sensor, clicking on the setup button in Figure 5 will appear below, and you can see the amount of the 16 channel analog input is converted to $+/-10 \mathrm{~V}$ or so, the sensor changes in the range of $85 \mathrm{~mm}$ to $45 \mathrm{~mm}$, You can see the obvious changes in the voltage value in Fig.7.

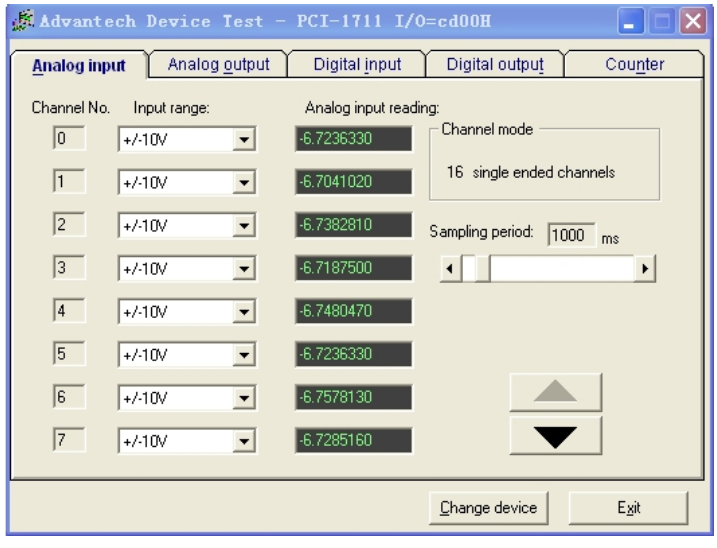

Fig.7. Analog input tab of the device test dialog

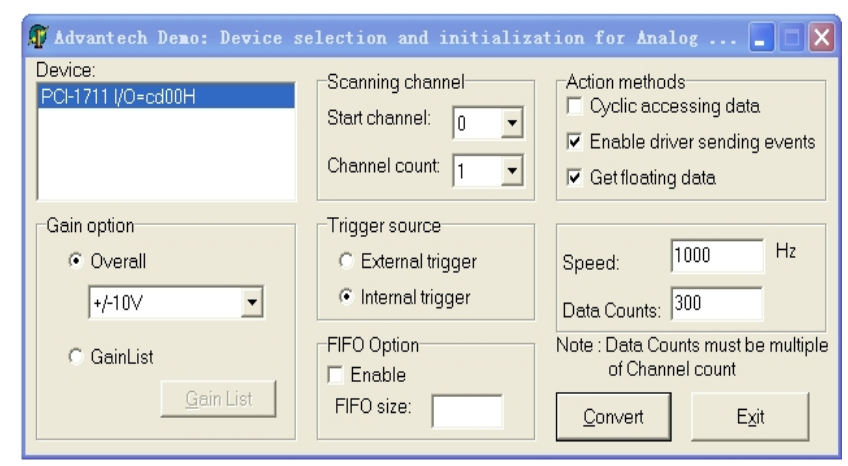

Fig.8. Setup dialog box

Find out the installation folder Advantech $\backslash$ Adsapi $\backslash$ Examples $\backslash$ Delphi $\backslash M A D \_I N T$, and run the exe inside the MAD_INT file, which is a multi channel AD conversion process. Specific parameters can be seen in Fig. 8 .

The above parameters can be used to set the starting channel, the total number of channels, sampling frequency, the number of data acquisition. Save settings, and click on the convert, appearing the results Fig.9.

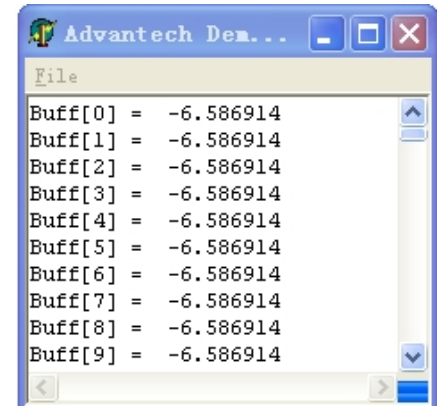

Fig.9. Device result of display dialog box

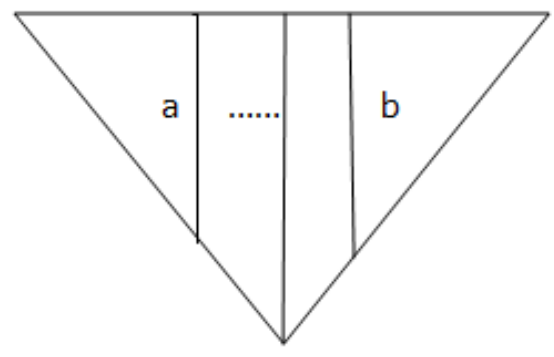

Fig.10. Sketch map of weld seam

The above data are saved as EXCEL form, which is stored in the column, and used in the calculation of the weld volume. The photoelectric sensor is measured in the vertical direction of the welding surface, and the height of the distance to the welding surface is constant. The welding surface is in the same horizontal plane along the vertical direction, The number of the measurements taken by the column is the same. Measure the end of a column, and move a few millimeters in the horizontal direction. When the weld is encountered, the measured value is greater than the value of the welding surface. Repeat the above work until the whole welding surface is finished. All of the data will be recorded in a table, and each column represents the value of the vertical direction of the weld. 


\section{Welding Data Processing}

The values are imported to MATLAB to calculate the weld volume, and then the two edges and the vertical direction of the welding are obtained using backward difference. Suppose that the shape of welding section is a triangle or trapezoid, the following is the algorithm and annotations described in MATLAB language.

$\mathrm{A}=$ data $\quad / /$ Import the excel table from the workspace and rename it to data, and assign it to A.

$\mathrm{A}=\operatorname{roundn}(\mathrm{A},-1) / /$ All the data in the table is accurate to a decimal point, accurate to $0.4 \mathrm{~mm}$.

$$
\text { for } \mathrm{j}=1: \operatorname{size}(A, 2)-1
$$

$B(:, j)=A(:, j+1)-A(:, j) ; / /$ The matrix $A$ to do backward difference, the difference is stored in the matrix B.

end

$\mathrm{j}=1$;

while $(B(:, \mathrm{j})==0)$

$\mathrm{j}=\mathrm{j}+1$;

$\mathrm{a}=\mathrm{j}$;

end // Recognition of the weld edge of the while loop statement.

$\mathrm{j}=\mathrm{a}$;

while $(B(:, j) \sim=0)$

$\mathrm{j}=\mathrm{j}+1$;

$\mathrm{b}=\mathrm{j}$;

end $\quad / /$ Second while to achieve the recognition of the other edge of the weld.

for $n=a: b-2$

$C(:, n-a+1)=A(:, n+1)-A(:, a) ; / / A$ difference between the value of the weld and the edge of the weld is done on the matrix $\mathrm{C}$

$\mathrm{E}=[]$;

$\mathrm{E}=\mathrm{abs}(\mathrm{C}) * 4 ; / /$ The voltage value is converted to the length, $4=(85-45) / 10$, the unit of $\mathrm{mm}$, and assigned to the $\mathrm{E}$.

End

$\mathrm{m}=\mathrm{a}$;

$\mathrm{V}(:, \mathrm{m}-\mathrm{a}+1)=0.5 * \mathrm{E}(:, \mathrm{m}-\mathrm{a}+1) * \mathrm{H}^{*} \mathrm{~L} ; / /$ The volume of the nearest MITSUBISHI column on the left side of the weld edge.

$\mathrm{V}(:, \mathrm{b}-\mathrm{a})=0.5 * \mathrm{E}(:, \mathrm{b}-\mathrm{a}-1) * \mathrm{H}^{*} \mathrm{~L} ; / /$ The right edge of the weld Mitsubishi column volume 
recently.

for $d=2: b-a-1$

$\mathrm{V}(:, \mathrm{d})=0.5^{*}[\mathrm{E}(:, \mathrm{d}-1)+\mathrm{E}(:, \mathrm{d})]^{*} \mathrm{H}^{*} \mathrm{~L} ; / /$ The middle of the trapezoidal cross section or rectangular volume.

end

$\mathrm{M}=\operatorname{sum}(\mathrm{V}(:))$; //The volume sum of matrix, obtained the weld volume

Note: $\mathrm{H}$ in the code refers to the distance between the horizontal direction and $\mathrm{L}$ the split length in vertical direction of the data being collected.

As shown in Figure 10, the volumes of $\mathrm{a}$ and $\mathrm{b}$ are the volume of the left and right sides of the algorithm in the MITSUBISHI column, respectively. The area of the middle section is calculated by the trapezoid. If the welding shape is not a triangle but a trapezoid, the middle section of the area is a rectangular, which is a special case of trapezoid and can treat consistently as a trapezoid. with the measured data we can have the following Fig. 11.

\section{$\left.\begin{array}{ll|l|l|l|l|l|l|l|l}1.049102 & 1.035156 & 1.694336 & 2.802734 & 3.173828 & 2.34375 & 1.225586 & 1.186523 & 1.225586\end{array}\right]$}

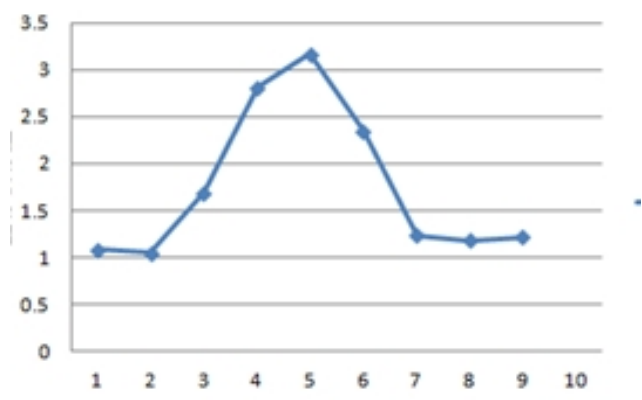

Fig.11. Measured value line chart

Figure 11 is the measured results detected in the horizontal direction of the weld $2 \mathrm{~mm}$ each time. The horizontal direction is $10 \mathrm{~mm}$, and the vertical direction is $298 \mathrm{~mm}$. Set up the collection of data per column as number 200, and the actual collection is 199 . The MATLAB program is used to obtain the volume $14328 \mathrm{~mm}^{3}$.

The volume of the weld joint is known, assume that the weld joint is filled in five times, by filling $1 / 25,3 / 25,1 / 5,7 / 25,9 / 25$ of the volume respectively. Given the density of steel is 7.9 $\mathrm{mg} / \mathrm{mm}^{3}$, we can find the corresponding volume value. And the filling mass of each time is $4.5 \mathrm{~g}$, $22.6 \mathrm{~g}, 31.7 \mathrm{~g}, 40.7 \mathrm{~g}$ and $13.5 \mathrm{~g}$. Given the outflow of molten steel welding speed is $1.2 \mathrm{~g} / \mathrm{s}$, the time of each welding is $11.3 \mathrm{~s}, 18.8 \mathrm{~s}, 3.8 \mathrm{~s}, 26.4 \mathrm{~s}, 33.9 \mathrm{~s}$.

Total volume $\mathrm{V}=14328 \mathrm{~mm}^{3}$, The resolution of the photoelectric sensor is $0.02 \mathrm{~mm}$.

Table 1. Accuracy calculation of robot intelligent metal welding

\begin{tabular}{|l|l|l|l|l|l|}
\hline V error & $\mathrm{V} 1=11.98\left(\mathrm{~mm}^{3}\right)$ & $\mathrm{V} 2=23.90\left(\mathrm{~mm}^{3}\right)$ & $\mathrm{V} 3=35.82\left(\mathrm{~mm}^{3}\right)$ & $\mathrm{V} 4=47.74\left(\mathrm{~mm}^{3}\right)$ & $\mathrm{V} 5=59.66\left(\mathrm{~mm}^{3}\right)$ \\
\hline V0 & $573.12\left(\mathrm{~mm}^{3}\right)$ & $2292.48\left(\mathrm{~mm}^{3}\right)$ & $5158.08\left(\mathrm{~mm}^{3}\right)$ & $9169.92\left(\mathrm{~mm}^{3}\right)$ & $14328\left(\mathrm{~mm}^{3}\right)$ \\
\hline $\begin{array}{l}\text { Percent } \\
\text { accuracy }\end{array}$ & $2.1 \%$ & $1.0 \%$ & $0.69 \%$ & $0.52 \%$ & $0.42 \%$ \\
\hline
\end{tabular}




\section{Conclusion}

According to Table1, the accuracy of robot intelligent metal welding can reach 2.5. The volume of the weld joint can be detected by using the photoelectric analog sensor, and the corresponding code is written according to the cross section of the weld joint. Errors are introduced in practice mainly by. Two reasons: The first one is when the welding surface and welding edge is not on the same plane, the measured data can not be imported directly into the program, instead we need a reference value, the value of the weld surface can be used instead of the value of the edge of the weld, conversely, too, otherwise the program will get an error. The other one is that the distance of each horizontal direction is limited, and it is not certain to be able to measure the lowest point of the weld. The measured data above deviates from the actual value. There are many other welding surface situations to study. For example, the welding surface is not in the same plane, the shape of the weld is irregular, etc. And we need to constantly improve the welding technology.

\section{References}

[1] Qian Donghai, Wang Xinfeng,Zhao Wei:Algorithm for the inverse kinematics calculation of 6DOF robots based on screw theory and Paden- Kahan sub- problems [J], Chinese Journal of Mechanical Engineering, Vol. 45, Issue 9, 2009,pp.72 - 76.

[2] Qiu Nin-jia, Shui Zhen, Li Ming-zhe,Zheng Cheng-xiang, Algorithm of 6 - DOF robot trajectory planning applied to special marking, Journal of Jili University. Vol.43, Issue 5, 2013,p.1308.

[3] Duan Hong-wei, Lan Hu, Zheng Hong-yan, Jian Xiao-xia, Image Processing of Robotic Vision Sensing for Typical Joints , Journal of Harbin university of science and Technology, 17, 4, 2012,pp. 37-41.

[4] Zou Yuanyuan, Zhao M ingyang, Zhang Lei, Gao Shiyi, Error analysis and structural analysis of structured light visual sensor for seam tracking, Chinese Journal of Scientific Instrumen, Vol. 29, Issue 12, 2008, pp. 2605-2610.

[5] Xie Zhi-meng, Gao Xiang-dong,Study and Prospect of Seam-tracking Technology Based on Vision Sensors, Transactions of the China Welding Institution, Vol. 4, 2005, pp. 5-9.

[6] Chang Shu-Hua, Song Yan-Qiu, Yang Yong-Min, Matching Preprocessing Technology of Binocular Stereo Vision, Journal of Jilin University, Vol. 50,Issue 1, 2012, pp. 1-10.

[7] Wen Qingchuan ,Da Feipeng, Fang Xu, Calibration for Gaze Tracking system Based on Binocular Stereo Vision, Actaopticasinica, Vol. 32, Issue 10, 2012,pp. 1-10.

[8] Huang Junfen, Jiang Lipei, The weld groove edge detection basedon two- dimensional wavelet analysis, Transactions of the China Welding Institution, Vol. 24, Issue 6, 2003, pp. 69-72.

[9] Zhen Heng-zhou, Tracking Control of Complex Seam in Laser Welding Using Visual Measurement System, Journal of Dalian university, Vol. 34,Issue 3, 2013, pp. 13-17.

[10] Hong bo,Wei Fuli,Lai Xin, A magnetic control arc sensor used in weld seam tracking',Transactions of the China Welding Institution, Vol. 29, Issue 5, 2008,pp. 1-4. 